Publ. Math. Debrecen (Submitted)

\title{
THE CONTRACTION PRINCIPLE IN EXTENDED CONTEXT
}

\author{
MIHÁLY BESSENYEI \\ Dedicated to the 60th birthday of Professor Zsolt Páles
}

\begin{abstract}
There are several extensions of the classical Banach Fixed Point Theorem in technical literature. A branch of generalizations replaces usual contractivity by weaker but still effective assumptions. Our note follows this stream, presenting an elementary proof for a known fixed point result. Some applications are also considered.
\end{abstract}

\section{INTRODUCTION}

Although the Contraction Principle appears partly in the method of successive approximation trough the works of Cauchy [8], Liouville [14], and Picard [16], its abstract and powerful version is due to Banach [1] and Cacciopoli [7]. This form of the Contraction Principle, quoted as the Banach Fixed Point Theorem, states that any contraction of a complete metric space has exactly one fixed point.

We can say that the Contraction Principle has made an explosion in contemporary mathematics. It has become the corner stone of Iterative Fixed Point Theory, has initiated new branches of important generalizations, and has found its legacy in many fields of mathematics via applications. The monographs by Berinde [2], by Granas and Dugundji [10], by Rus, Petruşel and Petruşel [18], and by Zeidler [20] give an excellent and detailed demonstration of this fact.

An important stream of the extensions of the Contraction Principle replaces contractivity by weaker but still effective properties. A rich overview of such generalizations can be found in the survey of Rhoades [17]. Among them, let us recall the pioneer works of Boyd and Wong [5], of Browder [6], and of Matkowski [15]. These investigations present fixed point results for nonlinear contractions. An other direction concerns linear quasicontractions, and was studied first by Ćirić [9] and also by Kannan [13]. For precise details, the Reader can have a quick look at the corollaries of this note. Surprisingly, applying (nonlinear) quasicontractions, the above mentioned cases get a common point of view: Independently and simultaneously, Hegedûs and Szilágyi [11] and Walter [19] have given fixed point results for this general setting.

These extensions have not merely theoretical importance, but enjoy many didactic and philosophical aspect, as well. For those ones, who read through and understand the various ideas, open a colorful perspective of the Contraction Principle.

The aim of the present note is to re-discover the theorem of Hegedús, Szilágyi and Walter, this wonderful and hidden treasure of Fixed Point Theory. Requiring a reasonable extra assumption on comparison functions, an elementary and self-contained approach can be given. This approach does not exceed the standard tools of classical analysis and the basic facts on metric spaces, and hence, according to our hope, might have some impact even in education.

Date: August 15, 2018.

2010 Mathematics Subject Classification. Primary 47H10; Secondary 54H25, 54A20, 54E25.

Key words and phrases. Banach Fixed Point Theorem, iteration, contraction principle, linear quasicontraction, nonlinar contraction, quasicontraction.

This research has been supported by the Hungarian Scientific Research Fund (OTKA) Grants K-111651. 


\section{NOTATIONS, NOTIONS, BASIC FACTS}

Throughout this note, $\mathbb{R}_{+}$and $\mathbb{N}$ stand for the set of nonnegative reals and natural numbers, respectively. If $f$ is a self-map of a nonempty set, then the composite iterates are defined via the usual recursion $f^{n+1}=f \circ f^{n}$ under the convention $f^{0}=\mathrm{id}$. The orbit and the double orbit induced by $f$ are defined in the next way:

$$
\mathcal{O}(x):=\left\{f^{n}(x) \mid n \in \mathbb{N} \cup\{0\}\right\} ; \quad \mathcal{O}(x, y):=\mathcal{O}(x) \cup \mathcal{O}(y) .
$$

Our investigations focus on nonlinear quasicontractions, when the distance of images is controlled by a given function of double orbits induced by the original points.

Definition. Under a comparison function we mean an increasing, upper semicontinuous function $\varphi: \mathbb{R}_{+} \rightarrow \mathbb{R}_{+}$which fulfills the properties $\varphi(0)=0$ and $\varphi(t)<t$ for $t>0$. Let $(X, d)$ be a metric space. A mapping $T: X \rightarrow X$ is called a weak quasicontraction with comparison function $\varphi$ (or briefly: a weak $\varphi$-quasicontraction) if it induces bounded orbits and, for all $x, y \in X$,

$$
d(T x, T y) \leq \varphi(\operatorname{diam} \mathcal{O}(x, y)) .
$$

Similarly, under a strong $\varphi$-quasicontraction we mean a mapping $T: X \rightarrow X$ fulfilling the property below for all $x, y \in X$ :

$$
d(T x, T y) \leq \varphi(\operatorname{diam}\{x, y, T x, T y\}) .
$$

Clearly, if a strong quasicontraction induces bounded orbits, then it is a weak quasicontraction. However, there exist strong quasicontractions that induce unbounded orbits [3], and hence they are not considered weak quasicontractions in our framework. Of course, the definition above subsumes the notions of linear quasicontractions and nonlinear contractions mentioned in the Introduction. The most unusual feature of a weak/strong quasicontraction is that its continuity cannot be guaranteed, even in the most simple cases [13]. This phenomenon makes impossible to apply the standard methods: New ideas have to be developed for establishing fixed point theorems.

Note that a weak quasicontraction can have at most one fixed point. Indeed, assume that $x_{0}$ and $y_{0}$ are distinct fixed points of a weak $\varphi$-quasicontraction $T$. Then, $d\left(x_{0}, y_{0}\right)>0$ and hence

$$
d\left(x_{0}, y_{0}\right)=d\left(T x_{0}, T y_{0}\right) \leq \varphi\left(\operatorname{diam} \mathcal{O}\left(x_{0}, y_{0}\right)\right)=\varphi\left(d\left(x_{0}, y_{0}\right)\right)<d\left(x_{0}, y_{0}\right) .
$$

A similar argument shows that the fixed point of a strong quasicontraction is unique provided that it exists at all.

In the sequel, let us present two less obvious properties for comparison functions and weak quasicontractions. The first one is well-known (see for example [3]). For the Readers' convenience, we sketch here the proof.

Lemma. The sequence of composite iterates of a comparison function tends to zero pointwise.

Proof. If $\varphi$ is a comparison function, then the limit property obviously holds at $t=0$. Fix $t>0$. If $\varphi(t)=0$, then $\varphi^{n}(t)=0$ for all $n \in \mathbb{N}$; if $\varphi(t) \neq 0$, then $\varphi^{2}(t)<\varphi(t)$ follows after iterating the inequality $\varphi(t)<t$. Proceeding by induction, we get that $\left(\varphi^{n}\right)$ is decreasing on $\mathbb{R}_{+}$. On the other hand, it is bounded from below. Therefore, $f=\lim _{n \rightarrow \infty} \varphi^{n}$ exists and takes nonnegative values. Assume indirectly that $f(t)>0$ for some $t>0$. Then, by upper semicontinuity, we arrive at

$$
f(t)=\lim _{n \rightarrow \infty} \varphi^{n+1}(t)=\limsup _{n \rightarrow \infty} \varphi\left(\varphi^{n}(t)\right) \leq \varphi\left(\limsup _{n \rightarrow \infty} \varphi^{n}(t)\right)=\varphi(f(t))<f(t) .
$$

This contradiction completes the proof.

Observe that monotonicity has played no role here. However, the proof of the next auxiliary lemma enlightens the importance of this property. 
Lemma. If $T$ is a weak $\varphi$-quasicontraction, then $T^{n}$ is a weak $\varphi^{n}$-quasicontraction.

Proof. Fix $k, l \in \mathbb{N}$ and let $T^{k} x, T^{l} y \in \mathcal{O}(x, y)$. Then the contractivity of $T$ and the monotonicity of $\varphi$ gives

$$
d\left(T^{k} x, T^{l} y\right)=d\left(T T^{k-1} x, T T^{l-1} y\right) \leq \varphi\left(\operatorname{diam} \mathcal{O}\left(T^{k-1} x, T^{l-1} y\right)\right) \leq \varphi(\operatorname{diam} \mathcal{O}(x, y)) .
$$

A similar argument leads to

$$
d\left(T^{k} x, T^{l} x\right) \leq \varphi\left(\operatorname{diam} \mathcal{O}\left(T^{k-1} x, T^{l-1} x\right)\right) \leq \varphi(\operatorname{diam} \mathcal{O}(x)) \leq \varphi(\operatorname{diam} \mathcal{O}(x, y)) .
$$

For $T^{k} y, T^{l} y \in \mathcal{O}(x, y)$, the same upper estimation can be obtained in the same way. That is, we have the next inequality between the double orbits of $\{x, y\}$ and $\{T x, T y\}$ :

$$
\operatorname{diam} \mathcal{O}(T x, T y)=\sup _{k, l \in \mathbb{N}}\left\{d\left(T^{k} x, T^{l} y\right), d\left(T^{k} x, T^{l} x\right), d\left(T^{k} y, T^{l} y\right)\right\} \leq \varphi(\operatorname{diam} \mathcal{O}(x, y)) .
$$

To complete the proof, we apply induction. For $n=1$, the statement holds trivially. Assume that it also remains true for some $n \in \mathbb{N}$. Then, applying the inequality above and the monotonicity of the comparison function,

$$
d\left(T^{n+1} x, T^{n+1} y\right)=d\left(T^{n} T x, T^{n} T y\right) \leq \varphi^{n}(\operatorname{diam} \mathcal{O}(T x, T y)) \leq \varphi^{n+1}(\operatorname{diam} \mathcal{O}(x, y))
$$

follows, which was to be proved.

Note also that an analogous statement cannot be formulated for strong quasicontractions. The reason for this, in particular, is that the iterates of a strong quasicontraction does not generate necessarily bounded orbits, even if the original mapping does.

\section{THE MAIN RESULT}

The main result of this note presents a fixed point theorem for weak quasicontractions of complete metric spaces. The proof has three stages. The first and standard one is devoted to show the Cauchy property of sequence of iterates. Then completeness gives the existence of a limit point. The forthcomings bring novelty: In the second step we show, that the iterates on the limit point tend also to this limit. In fact, it is an immediate consequence in the standard cases but now, in lack of continuity, will have a particular importance. The final step concludes that the orbit induced by the limit has zero diameter, which is the desired fixed point property. The technical lemmas of the previous section play a key role in the arguments.

Theorem. Any weak quasicontraction of a complete metric space has a unique fixed point. Moreover, the sequence of iterates at any point converges to this fixed point.

Proof. Let $(X, d)$ complete metric space and let $T: X \rightarrow X$ be a weak $\varphi$-quasicontraction. Fix $x \in X$ arbitrarily. We show that $\left(T^{n} x\right)_{n=0}^{\infty}$ has the Cauchy property. The boundedness of orbits and the pointwise convergence property of $\left(\varphi^{n}\right)$ provides that, for all $\varepsilon>0$, there exists $n_{0} \in \mathbb{N}$ such that $\varphi^{n_{0}}(\operatorname{diam} \mathcal{O}(x))<\varepsilon / 2$. If $n>n_{0}$, then

$$
d\left(T^{n_{0}} x, T^{n} x\right) \leq \varphi^{n_{0}}\left(\operatorname{diam} \mathcal{O}\left(x, T^{n-n_{0}} x\right)\right)=\varphi^{n_{0}}(\operatorname{diam} \mathcal{O}(x))<\varepsilon / 2 .
$$

Hence the use of the triangle inequality leads to $d\left(x_{n}, x_{m}\right)<\varepsilon$ for all $n, m>n_{0}$. In other words, the iterates of $x$ defines a Cauchy sequence.

The completeness guarantees the existence of $x_{0} \in X$ such that $T^{n} x \rightarrow x_{0}$. Our claim is that $T^{n} x_{0} \rightarrow x_{0}$ also holds. Indeed,

$$
d\left(x_{0}, T^{n} x_{0}\right) \leq d\left(x_{0}, T^{n} x\right)+d\left(T^{n} x, T^{n} x_{0}\right) \leq d\left(x_{0}, T^{n} x\right)+\varphi^{n}\left(\operatorname{diam} \mathcal{O}\left(x, x_{0}\right)\right) \rightarrow 0
$$

as $n \rightarrow \infty$. 
To complete the proof, we suffice to show that diam $\mathcal{O}\left(x_{0}\right)=0$. Suppose to the contrary that this is not the case. Then, for all $n, k \in \mathbb{N}$, we arrive at

$$
d\left(T^{n} x_{0}, T^{n+k} x_{0}\right) \leq \varphi^{n}\left(\operatorname{diam} \mathcal{O}\left(x_{0}, T^{k} x_{0}\right)\right)=\varphi^{n}\left(\operatorname{diam} \mathcal{O}\left(x_{0}\right)\right) \leq \varphi\left(\operatorname{diam} \mathcal{O}\left(x_{0}\right)\right) .
$$

Therefore,

$$
\sup _{n, m \in \mathbb{N}} d\left(T^{n} x_{0}, T^{m} x_{0}\right) \leq \varphi\left(\operatorname{diam} \mathcal{O}\left(x_{0}\right)\right)<\operatorname{diam} \mathcal{O}\left(x_{0}\right)
$$

yielding

$$
\operatorname{diam} \mathcal{O}\left(x_{0}\right)=\sup _{n \in \mathbb{N}} d\left(x_{0}, T^{n} x_{0}\right) .
$$

On the other hand, $T^{n} x_{0} \rightarrow x_{0}$. This property, together with the above one, implies that there exists some $n_{0} \in \mathbb{N}$ such that

$$
\operatorname{diam} \mathcal{O}\left(x_{0}\right)=\max \left\{d\left(x_{0}, T^{k} x_{0}\right) \mid k=1, \ldots, n_{0}\right\}
$$

Let $k \in\left\{1, \ldots, n_{0}\right\}$ be the index via the diameter is represented. Then, for all $n \in \mathbb{N}$, we have

$$
\begin{aligned}
d\left(x_{0}, T^{k} x_{0}\right) & \leq d\left(x_{0}, T^{n+k} x_{0}\right)+d\left(T^{n+k} x_{0}, T^{k} x_{0}\right) \\
& \leq d\left(x_{0}, T^{n+k} x_{0}\right)+\varphi^{k}\left(\operatorname{diam} \mathcal{O}\left(T^{n} x_{0}, x_{0}\right)\right) \\
& =d\left(x_{0}, T^{n+k} x_{0}\right)+\varphi^{k}\left(\operatorname{diam} \mathcal{O}\left(x_{0}\right)\right) \\
& \leq d\left(x_{0}, T^{n+k} x_{0}\right)+\varphi\left(\operatorname{diam} \mathcal{O}\left(x_{0}\right)\right) .
\end{aligned}
$$

Passing the limit,

$$
\operatorname{diam} \mathcal{O}\left(x_{0}\right)=d\left(x_{0}, T^{k} x_{0}\right) \leq \varphi\left(\operatorname{diam} \mathcal{O}\left(x_{0}\right)\right)<\operatorname{diam} \mathcal{O}\left(x_{0}\right)
$$

This contradiction completes the proof of the first statement. The second statement is obvious.

Not claiming completeness, we demonstrate the efficiency of the main result in form of alternative approaches to known fixed point results. The first one concerns linear quasicontractions of Ćirić-type [9], while the second one nonlinear contractions studied by Boyd and Wong [5], by Browder [6], and by Matkowski [15]. The Banach Fixed Point Theorem is not detailed, since it is an immediate consequence of both corollaries.

Corollary. If $(X, d)$ is a complete metric space, $q \in] 0,1[$ is fixed, and $T: X \rightarrow X$ satisfies

$$
d(T x, T y) \leq q \operatorname{diam}\{x, y, T x, T y\}
$$

for all $x, y \in X$, then $T$ has a unique fixed point. Moreover, the sequence $\left(T^{n} x\right)$ converges to the fixed point for all $x \in X$.

Hint. Obviously, $T$ is a strong quasicontraction with comparison function $\varphi(t)=q t$. Therefore, in view of the main result, one should check only the boundedness of orbits. For any $x \in X$, consider its $n$-orbit

$$
\mathcal{O}_{n}(x)=\left\{T^{k} x \mid k=0,1, \ldots, n\right\}
$$

Then,

$$
\operatorname{diam} \mathcal{O}_{n}(x) \leq d(x, T x)+\operatorname{diam} \mathcal{O}_{n-1}(T x) \leq d(x, T x)+q \operatorname{diam} \mathcal{O}_{n}(x) .
$$

The arranged form of this inequality shows that $n$-orbits are uniformly bounded. That is, the entire orbit is bounded, as well. 
Corollary. If $(X, d)$ is a complete metric space, $\varphi$ is a comparison function, and $T: X \rightarrow X$ satisfies

$$
d(T x, T y) \leq \varphi(d(x, y))
$$

for all $x, y \in X$, then $T$ has a unique fixed point. Moreover, the sequence $\left(T^{n} x\right)$ converges to the fixed point for all $x \in X$.

Hint. We shall concentrate again on boundedness of orbits. For this, we use the original (and beautiful) idea of domain invariance: If $T$ makes small perturbation on the center of a ball, then maps the ball into itself. Let $p \in X$ and $r>0$ be fixed. If $q \in B(p, r)$, then

$$
d(p, T q) \leq d(p, T p)+d(T p, T q) \leq d(p, T p)+\varphi(d(p, q))<r
$$

provided that $d(p, T p)<r-\varphi(r)$ holds. That is, $T q \in B(p, r)$. In particular, $d(p, T p)<r$; substituting $q=T p$, we arrive at $T^{2} p \in B(p, r)$. Applying induction, $T^{m} p \in B(p, r)$ follows for all $m \in \mathbb{N}$.

On the other hand, due to the auxiliary lemmas, $d\left(T^{n} x, T^{n+1} x\right) \rightarrow 0$ for all $x \in X$. The domain invariance ensures that $T^{n+m} x \in B\left(T^{n} x, r\right)$ for all $m \in \mathbb{N}$ with a sufficiently large index $n$. In other words, our mapping induces bounded orbits.

Unfortunately, a direct and common generalization of the previous corollaries cannot be given for arbitrary strong quasicontractions. This phenomenon is related to our earlier comment concerning strong quasicontractions that are not weak ones, since they induces unbounded orbits. Among these kind of strong quasicontractions, there exist fixed point free ones. However, under an extra assumption on the comparison function, this problem can be avoided, and the next result is obtained. Note that is also covers the case of Banach. For details of the proof, consult [3].

Corollary. If $(X, d)$ is a complete metric space, $\varphi$ is a comparison function such that $\varphi^{n}(t) \leq c_{n} t$ holds with some convergent series $\sum c_{n}$, then any strong $\varphi$-quasicontraction has a unique fixed point. Moreover, the sequence of iterates converges pointwise to this fixed point.

An other straightforward consequence of the main result is that any weak or strong quasicontraction of a compact metric space has a unique fixed point. This can be considered as a counterpart of the statement on strictly nonexpansive mappings of compact metric spaces.

Finally, let us mention two possibilities on further research. First, as it is well known, the Banach Fixed Point Theorem has a particular importance in Fractal Theory (see the paper of Hutchinson [12]). A natural question is, what kind of new impact has the main result in this field? Second, that relaxing the properties of the embedding space is intensively investigated in present technical literature. For example, the Matkowski Fixed Point Theorem remains true in so-called regular semimetric spaces [4]. It is an open problem, whether the main result can be replaced into this general context or not?

The main theorem points out that boundedness of orbits has more importance then the classical approaches suggest. We should say that it is a well-established assumption: provides brief form and effective applications simultaneously. In our opinion, it gives certainly a deeper understanding of the well-known Contraction Principle.

\section{REFERENCES}

[1] S. Banach, Sur les opérations dans les ensembles abstraits et leur application aux équations intégrales, Fund. Math. 3 (1922), 133-181.

[2] V. Berinde, Iterative approximation of fixed points, second ed., Lecture Notes in Mathematics, vol. 1912, Springer, Berlin, 2007.

[3] M. Bessenyei, Nonlinear quasicontractions in complete metric spaces, Expo. Math. 33 (2015), 517-525. 
[4] M. Bessenyei and Zs. Páles, A contraction principle in semimetric spaces, J. Nonlinear Convex Anal. (2016), to appear.

[5] D. W. Boyd and J. S. W. Wong, On nonlinear contractions, Proc. Amer. Math. Soc. 20 (1969), $458-464$.

[6] F. E. Browder, On the convergence of successive approximations for nonlinear functional equations, Indag. Math. 30 (1968), 27-35.

[7] R. Caccioppoli, Un teorema generalle sulla esistenza di elementi uniti in una transformazione funzionale, Rend. Acc. Naz. Lincei 11 (1930), 794-799.

[8] A. Cauchy, Oeuvres completes X, Gauthier-Villars, Paris, 1835/1958.

[9] Lj. B. Ćirić, A generalization of banach's contraction principle, Proc. Amer. Math. Soc. 45 (1974), $267-273$.

[10] A. Granas and J. Dugundji, Fixed point theory, Springer Monographs in Mathematics, Springer-Verlag, New York, 2003.

[11] M. Hegedús and T. Szilágyi, Equivalent conditions and a new fixed point theorem in the theory of contractive type mappings, Math. Japon. 25 (1980), 147-157.

[12] J. E. Hutchinson, Fractals and self-similarity, Indiana Univ. Math. J. 30 (1981), no. 5, 713-747.

[13] R. Kannan, Some results on fixed points. II, Amer. Math. Monthly 76 (1969), 405-408.

[14] J. Liouville, Sur le développement des fonctions ou parties de fonctions en séries, etc., Second Mémoire J. Math. 2 (1837), 16-35.

[15] J. Matkowski, Integrable solutions of functional equations, Dissertationes Math. 127 (1975), 1-68.

[16] E. Picard, Mémoire sur la théorie des équations aux dérivée partielles et la méthode des approximations successives, J. Math. Pures. et Appl. 6 (1890), 145-210.

[17] B. E. Rhoades, A comparison of various definitions of contractive mappings, Trans. Amer. Math. Soc. 226 (1977), 257-290.

[18] I. A. Rus, A. Petruşel, and G. Petruşel, Fixed point theory, Cluj University Press, Cluj-Napoca, 2008.

[19] W. Walter, Remarks on a paper by F. Browder about contraction, Nonlinear Anal. 5 (1981), 21-25.

[20] E. Zeidler, Nonlinear functional analysis and its applications. I, Springer-Verlag, New York, 1986, Fixed-point theorems.

Institute of Mathematics, University of Debrecen, H-4010 Debrecen, Pf. 12, Hungary

E-mail address: bessed science . unideb.hu 